\title{
Cryptococcal Meningitis
}

\author{
R. MCDONALD, E. N. GREENBERG, and R. KRAMER \\ From the Department of Paediatrics, University of Cape Town, and \\ Groote Schuur Hospital, Observatory, Cape, South Africa
}

\begin{abstract}
McDonald, R., Greenberg, E. N., and Kramer, R. (1970). Archives of Disease in Childhood, 45, 417. Cryptococcal meningitis. A report is given of 3 children who suffered from cryptococcal meningitis. The prognosis of the disease has improved, as effective antifungal agents have become available. Two of our cases recovered after prolonged treatment with amphotericin B and 5-fluorocytosine, respectively. The latter drug has the advantage of oral administration.
\end{abstract}

Cryptococcal meningitis in children is uncommon and used to have a high mortality rate. It occurs either as a primary illness, or secondarily in patients on immunosuppressants or long-term corticosteroids (Littman and Walter, 1968). Neuhauser and Tucker (1948) described the radiological changes in the lungs, as well as intracranial calcification, in 3 infants with cryptococcosis, all of whom were under 2 months of age. These, as well as a few other cases reported in the very young, were probably either congenital or else acquired during delivery.

There was no satisfactory drug therapy before 1956. Sulphonamides and antibiotics were of no value, while corticosteroids actually exacerbated lesions in laboratory animals (Pimstone and Saunders, 1959). A preparation known as cycloheximide (Actidione) had very good antifungal effects in vitro, but in human infections with $C$. neoformans it proved of little value (Carton, 1952; Pimstone and Saunders, 1959). The introduction of amphotericin B in 1956 was a big advance, and reports of its use in adults (Rippey et al., 1965; Keall, Wilkie, and Binns, 1968) and in children (Emanuel et al., 1961; Siewers and Cramblett, 1964) soon appeared. A new drug, 5-fluorocytosine, is being tried and there have been publications from the United States (Tassel and Madoff, 1968) and Great Britain (Watkins et al., 1969) on its successful use in adults with systemic fungal disease, but there have been no previous reports of its use in children.

In the past 14 years we have seen 3 children with cryptococcal meningitis and their case histories are given to indicate the manner in which the disease

Received 23 September 1969. may show itself and the improved present-day prognosis.

\section{Case Reports}

Case 1. A girl of 4 years was admitted from her home in the country in August 1955 because of an increasingly severe headache for 2 months. She had been vomiting for 3 weeks and had become drowsy and confused in the last 3 days before coming into hospital.

She was apathetic with a high temperature which sometimes reached $40^{\circ} \mathrm{C}$. Neck stiffness was present, and a cracked pot sound on percussion of the skull. Her pupils were dilated, reacting poorly to light and there was bilateral papilloedema. There was a right facial weakness. ESR $78 \mathrm{~mm} . / 1 \mathrm{hr}$. To relieve the raised intracranial pressure, burr holes were made into the skull and catheter drainage employed. Exploration of the posterior fossa was made but no evidence of a space-occupying lesion was found. CSF protein level was increased, and the sugar and chloride both substantially decreased, with a large number of cells, chiefly lymphocytes; no organisms were seen on direct smear or culture. It was thought that the condition might be tuberculous meningitis and anti-tuberculosis therapy was started, but without effect. Numerous examinations of the CSF continued to show a raised protein, with sugar usually decreased and varying numbers of lymphocytes and polymorphs. On 12 October, nearly 7 weeks after the child's admission, C. neoformans was grown on culture of the CSF, though none had been seen on the direct smear. By now the child was in a state of spastic quadriplegia and had bilateral optic atrophy. Actidione was then given for 2 weeks, but cryptococci continued to be found in the CSF and the child did not improve. Eventually, after 3 months in hospital, at the parents' request she was transferred to a hospital nearer her home and it is unlikely that she lived much longer.

Comment. This child presented with signs of increased intracranial pressure. The CSF was infected but no organisms were grown from it for several weeks 
when cryptococci were eventually cultured. Actidione failed to effect any improvement and it is doubtful if the child could have been saved, even with present-day therapy, as the illness was already far advanced when the causative diagnosis was made.

Case 2. A boy of 4 years, who also lived in the country, was admitted in June 1966. He had first been taken to another hospital for abdominal pain and fever. As the latter persisted and he had become drowsy, he was referred to us. He looked ill but was fully conscious, with neck stiffness, photophobia, and bilateral papilloedema. CSF protein $35 \mathrm{mg} . / 100 \mathrm{ml}$., sugar decreased, but chloride normal; 100 cells/cu.mm., all polymorphs; no organisms seen on direct smear or culture. Next day another lumbar puncture showed that all the cells, $242 / \mathrm{cu} . \mathrm{mm}$., were lymphocytes, and India ink stain showed up moderate numbers of cryptococci. These were found to be virulent on intracerebral inoculation of mice. Amphotericin B was then given intravenously for 6 out of every 24 hours and continued daily for 6 weeks. No toxic effects on bone-marrow were seen, but fever continued during the whole period of treatment. During this time 4 further lumbar punctures were done and cryptococci were seen every time. After stopping drug therapy the temperature fell to normal and the child's condition remained good for the remainder of his stay in hospital. Lumbar punctures were performed on 2 occasions after treatment had been stopped and showed that CSF protein was still somewhat raised at $70 \mathrm{mg} .100 \mathrm{ml}$., but the lymphocytes had fallen to under $25 / \mathrm{cu}$.mm.; cryptococci were still seen but did not grow on culture. After $3 \frac{1}{2}$ months in hospital the boy was sent home. One month later he was asymptomatic, but a few cryptococci were still seen in the CSF. We have not seen him since 1966, but he is reported (July 1969) to remain well.

Comment. This patient was seen earlier in the course of the disease than the first one, but had been ill for over 2 weeks. Cryptococci were not seen at the first lumbar puncture, but, because of the long duration of symptoms, another CSF examination was made after 24 hours, when the organisms were both seen and cultured. Amphotericin B was by now available and the child made a good recovery without serious toxic effects from the drug.

Case 3. A girl of 7 years came to us from her home on a farm in the country in May 1969. She had been complaining for 3 months of a headache which was progressively getting worse and ultimately had become very severe. For 2 days she had been feverish, with anorexia, vomiting, and photophobia. On the day she was brought to hospital she had 4 generalized convulsions. On arrival she was conscious though irritable and with intense headache. Her neck was stiff but no papilloedema was present. CSF protein $100 \mathrm{mg}$./ $100 \mathrm{ml}$., sugar absent, and chloride $114 \mathrm{mEq} / \mathrm{l}$; there were 360 polymorphs, 285 lymphocytes, and 85 monocytes/cu.mm., and cryptococci were seen on the smear when this was stained with India ink. 5-fluorocytosine $500 \mathrm{mg}$. was given 8 -hourly by mouth for the next $4 \frac{1}{2}$ weeks. By the second day the headache was already getting better and photophobia was less severe, and from then on she made steady progress. Within a few days all symptoms had gone and she was moving freely around her bed. The temperature, however, remained high and after $4 \frac{1}{2}$ weeks the drug dosage was increased to $500 \mathrm{mg}$. every 6 hours, a total dose of $2 \mathrm{~g}$. per day. Whether due to the increased dose or not, the temperature gradually fell to normal by the 9th hospital week. 5-fluorocytosine was continued for 10 weeks. A high ESR is said to occur when 5-fluorocytosine is used. At the start our patient's ESR level was $41 \mathrm{~mm}$./hour, by the 4 th week this had risen to 72 but thereafter it steadily fell, reaching 15 $\mathrm{mm}$./hour by the 8 th week. There was no disturbance of white cells or platelets during therapy. Blood urea and creatinine levels at the beginning and end of treatment remained normal. SGOT was reported as 60 units on 2 occasions, but on 4 other tests was always below 40 units. After 1 month, well-marked finger clubbing was noted, but this gradually decreased and by the time of discharge had nearly disappeared. Cryptococci were seen and grown on 7 and 20 May 1969. Thereafter there was no growth, though scanty organisms were seen from time to time. A slight increase of CSF protein persisted at about $70 \mathrm{mg} .100 \mathrm{ml}$. After 3 months the child was sent home, completely symptom free. One month after discharge she was well; CSF protein was $20 \mathrm{mg} . / 100 \mathrm{ml}$, , no cells or cryptococci being seen.

Comment. This child arrived at hospital with severe symptoms present for 3 months. She was, however, fully conscious and had no papilloedema. Treatment with 5-fluorocytosine given by mouth resulted in a rapid and satisfactory response, and no ill-effects were encountered.

\section{Discussion}

The primary site of cryptococcal infection in man is the respiratory tract and from there dissemination is blood borne. Clinical manifestations are pulmonary or neurological. The disease is characterized by its insidious onset, and chronic and usually fatal course, though remissions have been reported even without specific antifungal treatment. When the central nervous system is involved, the result may be meningitis or meningoencephalitis, while on some occasions a granulomatous lesion of cerebrum or cerebellum may give rise to symptoms and signs suggesting a space-occupying mass.

The main source of cryptococci has been found to be in the excreta of pigeons and, to a much lesser extent, excreta of other birds. Pigeon handlers are said to have a much higher level of antibodies to cryptococci than non-handlers (Littman and Walter, 1968). Contaminated milk has also been incriminated as a source of infection.

$C$. neoformans is an encapsulated yeast which is 
detected by staining with India ink, which shows up the non-staining capsule around the organism. Without this means of identification, the cryptococci may be mistaken for lymphocytes or other mononuclear cells. The best growth is on Sabouraud's medium, but this is slow and cultures should not be discarded for several weeks. Pathogenicity is assessed by intracerebral inoculation of mice, who become ill within 6 weeks if the infection is a virulent one.

At the onset of the illness there is minimal constitutional disturbance. There may be some fever with cough, loss of weight, and dyspnoea on exertion in the pulmonary type. The $x$-ray may show a solitary lesion, pneumonic infiltration, or widespread dissemination, resembling miliary tuberculosis. If the central nervous system is affected the signs may be those of a meningitis or a spaceoccupying lesion as in our first patient. The CSF has increased protein, decreased sugar, and a lymphocytic pleocytosis. If untreated there is usually progressive deterioration, though death may be delayed for several months.

The introduction of amphotericin B has greatly improved the outlook. It is usually given in a daily dose of $0.5-1 \mathrm{mg} . / \mathrm{kg}$. intravenously, though some would start with an even smaller dose. It is given over part of each day, if the patient can tolerate it, otherwise at longer intervals. Intrathecal injections have occasionally been resorted to when intravenous therapy has been ineffective (Littman and Walter, 1968). Oral forms of the drug exist, but according to Butler (1966), the colloidal form though adequately absorbed gives rise to severe abdominal cramps, diarrhoea, nausea, and anorexia. The other, microcrystalline form, has no ill-effects, but does not reach adequate blood levels.

Emanuel et al. (1961) gave an account of a child treated with amphotericin B who made a complete recovery from cryptococcal meningitis. The patient was known to have been playing in an area where pigeon excreta were abundant. At that time the authors had been able to trace only 23 cases of cryptococcal meningitis in children under 15 years. Only 5 had survived, and 3 had neurological sequelae. All had occurred before amphotericin B was available. Siewers and Cramblett (1964) described 4 children with cryptococcal disease, 2 of whom had meningitis. They were treated with amphotericin $B$ and both recovered.

Though effective, amphotericin $\mathrm{B}$ has to be given intravenously, and over a long period this is something of a disadvantage. In addition, a number of toxic effects have been reported (Emanuel et al., 1961; Siewers and Cramblett, 1964; Rippey et al.,
1965; Keall et al., 1968). These include nausea, vomiting, fever, headache, anaemia, alteration of liver function, and local phlebitis. The most serious, however, is disturbance of renal function with raised blood urea and creatinine, and with proteinuria, red and white blood cells, and casts in the urine. Butler (1966) stated that over $80 \%$ of patients have renal abnormalities and that no way to prevent this has been found. Decreased glomerular filtration rate and tubular lesions have also been reported. Persistence of renal disorder is apparently related to total dosage and may be lessened by increasing the time interval between doses. In Butler's report, which came from the National Institutes of Health, Bethesda, U.S.A., it has been found that of 47 patients treated for cryptococcal meningitis with amphotericin $\mathrm{B}, 41$ showed initial improvement with disappearance of cryptococci from the CSF, but that relapses, from 1 to 4 times each, occurred in 11 . This left 30 who responded favourably from the start, while 6 died.

There have been few reports on the use of 5fluorocytosine as yet. Tassel and Madoff (1968) reported a good response in an elderly patient with meningitis who had relapsed after initial improvement on amphotericin B. In another case (Watkins et al., 1969), the patient could not tolerate amphotericin $B$, but improved rapidly on 5-fluorocytosine. The big advantage of this drug is that it is given by mouth, and high CSF levels can be attained. Toxic effects are not yet fully documented, but depression of blood-forming elements in the bonemarrow should be looked for as well as alteration in liver functions. In our patient, no abnormal haematological effects were seen and while the SGOT was a little raised on 2 occasions, for the most part this was normal. No disturbance of kidney function was observed.

We do not know the optimal dose for children, but that given to our patient seemed satisfactory, even when the dose was increased. In our case, duration of therapy was determined by exhaustion of supplies after 10 weeks. By that time only occasional cryptococci could be found in the CSF and there had been no growth on culture for many weeks. As the disease is a chronic one, with a marked tendency to relapse, 10 weeks' therapy does not seem to us to be excessive. Whether our last patient will still relapse remains to be seen, but we are encouraged by the fact that our second patient, who received amphotericin $B$, and who still showed scanty cryptococci in the CSF on his discharge from hospital in 1966, is alive and well 3 years later without any further treatment. 
Dr. J. G. Burger, Superintendent of Groote Schuur Hospital, gave permission to publish this report. Thanks are due to Professor L. Eales for making a supply of 5-fluorocytosine available to us for our third patient. The drug had been supplied by Roche Products Ltd. for trial purposes.

\section{REFERENCES}

Butler, W. T. (1966). Fharmacology, toxicity, and therapeutic usefulness of amphotericin B. Fournal of the American Medical Association, 195, 371.

Carton, C. A. (1952). Treatment of central nervous system cryptococcosis. Annals of Internal Medicine, 37, 123.

Emanuel, B., Ching, E., Lieberman, A. B., and Goldin, M. (1961). Cryptococcus meningitis in a child successfully treated with Amphotericin B, with a review of the pediatric literature. Fournal of Pediatrics, 59, 577.

Keall, D. D., Wilkie, J. A., and Binns, C. (1968). Cryptococcosis: a survey of cases diagnosed in Western Australia, with a report of 2 cases. Medical Fournal of Australia, 2, 665.

Littman, M. L., and Walter, J. E. (1968). Cryptococcosis: current status. American Fournal of Medicine, 45, 922.
Neuhauser, E. B. D., and Tucker, A. (1948). The roentgen changes produced by diffuse torulosis in the newborn. American Fournal of Roentgenology, 59, 805.

Pimstone, B. L., and Saunders, S. J. (1959). Aspects of cryptococcosis. South African fournal of Laboratory and Clinical Medicine, 5, 156.

Rippey, J. J., Roper, W. A. G., Jeanes, A. L., and Bright, M. V. (1965). Cryptococcal meningo-encephalitis. Fournal of Clinical Pathology, 18, 296.

Siewers, C. M. F., and Cramblett, H. G. (1964). Cryptococcosis (torulosis) in children. Pediatrics, 34, 393.

Tassel, D., and Madoff, M. A. (1968). Treatment of candida sepsis and cryptococcus meningitis with 5-fluorocytosine. Fournal of the American Medical Association, 206, 830.

Watkins, J. S., Campbell, M. J., Gardner-Medwin, D., Ingham, H. R., and Murray, I. G. (1969). Two cases of cryptococcal meningitis, one treated with 5-fluorocytosine. British Medical Fournal, 3, 29.

Correspondence to Dr. R. McDonald, Department of Paediatrics and Child Health, University of Cape Town, Observatory, Cape, South Africa. 